\title{
Characteristics of secondary electron images from in-lens and conventional Everhart-Thornley detectors - evidence for the energy-based differentiation of high resolution SE1 and delocalized SE2 signals.
}

\author{
B. J. Griffin ${ }^{1}$, D.C. Joy ${ }^{2}$ and J. R. Michael ${ }^{3}$
}

1 Centre for Microscopy, Characterization and Analysis, The University of Western Australia, Crawley, WA Australia 6009

2 Center for NanoPhase Materials Science, Oak Ridge National Laboratory, Oak Ridge, TN 37831

3 Sandia National Laboratories, PO Box 5800, Albuquerque, NM 87185-0886

Remarkable differences in secondary electron images have been consistently observed in a number of samples when switching between a Zeiss in-lens detector (INL), FEI through-the-lens detector (TLD) and their respectively below-lens conventional Everhart-Thornley (E-T) SED (fig. 1). The differences between images are not simply due to the known variations in detector efficiencies or image $\mathrm{S} / \mathrm{N}$ as signal line profiles show (fig. 2).

Recent electron spectroscopy has shown that where a beam booster is employed to attract SE to the Zeiss INL SED the SE signal to the below-lens E-T SED is strongly filtered and dominated by higher energy SE relating to the BSE, to give a material contrast-rich E-T SED image [1]. An important implication of this study is that the high resolution SE1 arising from primary beam impact are lower in energy, at least on average, than those SE2 generated by the delocalized BSE leaving the sample. This data is supported by our imaging and SE intensity measurements obtained by varying the deflector "mirror" voltage of a TLD on a FEI Helios FESEM (fig. 3).

The previous data, however, fail to explain the material contrast-rich consistency of E-T SED images across a range of old and new instruments that lack a beam booster or similar configuration.

It is suggested here that the collector cage of most, if not all, E-T SED acts to filter out this SE1 signal, by trapping the low energy component on the wires of the cage. Consequently the E-T SED is incapable of providing true surface detail at other than low accelerating voltages, where the electron range is suitable short and this information is contained in the SE2 signal. In summary, our current view is that the conventional E-T SED is, in usual practice, a "SE2" detector whereas the inlens SED variants collect a wider energy range, encompassing the lower energy SE1 component, and so provides images of better $\mathrm{S} / \mathrm{N}$, resolution and surface-related information.

We have found that E-T SED images do become enriched in SE1 detail with increasing working distance. The INL contrast can be matched at $50 \mathrm{~mm}$ WD by inverting and subtracting a suitably scaled BSE image from the E-T SED image (fig. 4). The longer path length to the SED allows greater acceleration of the emitted SE resulting in a better collection of lower energy SE.

The collection energy ranges for SED and SE1/SE2 signals is schematically illustrated in figure 5. A below-lens SED designed to efficiently collect the low energy SE1 should provide all SEM with the ability to image at near the probe size, a $\times 2-3$ fold resolution improvement, and to image the surface state of the sample. 
[1] K. Kumagai, \& T. Sekiguchi, Ultramicroscopy (2009) accepted, on-line.

[2] Len Green, Adelaide Microscopy, is acknowledged for expert support on the Helios instrument. Support of the AMMRF is also acknowledged.
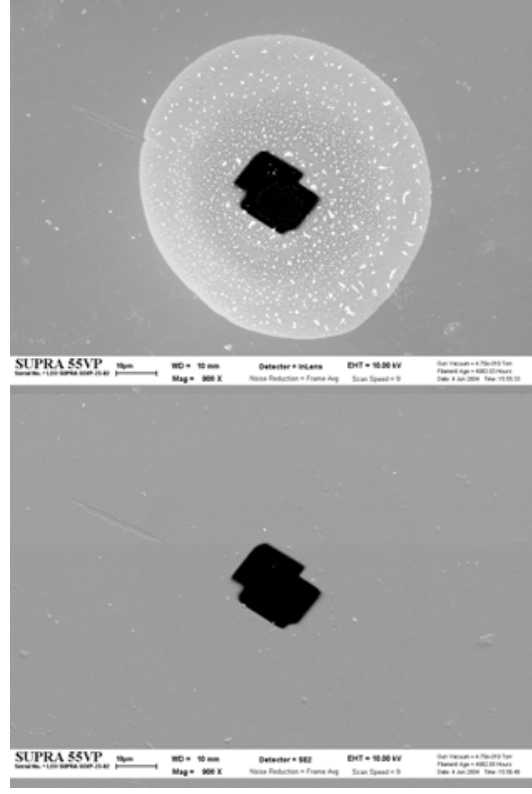

Figure 1(a): In-lens (top) and E-T (bottom) SEI of an Au-coated diamond around an area sputtered by the $\mathrm{Cs}^{+}$ beam of a N50 ion probe. $E_{o}=10 \mathrm{kV}$. Cs spatter is visible in the INL SEI.

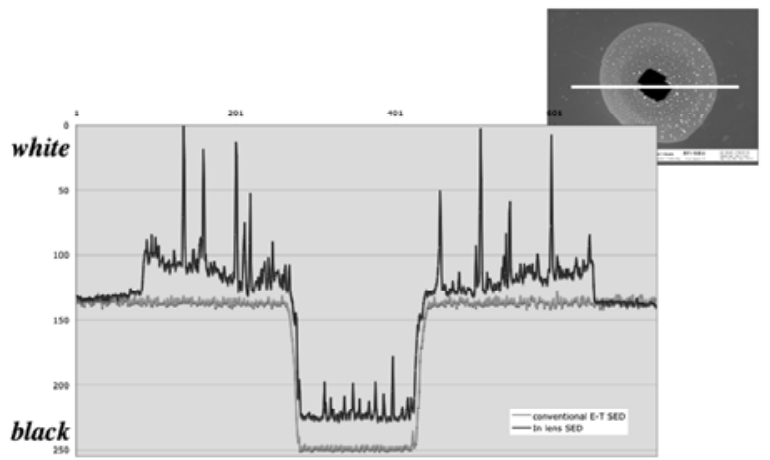

Figure 2: Signal profiles from images in fig.1(a).

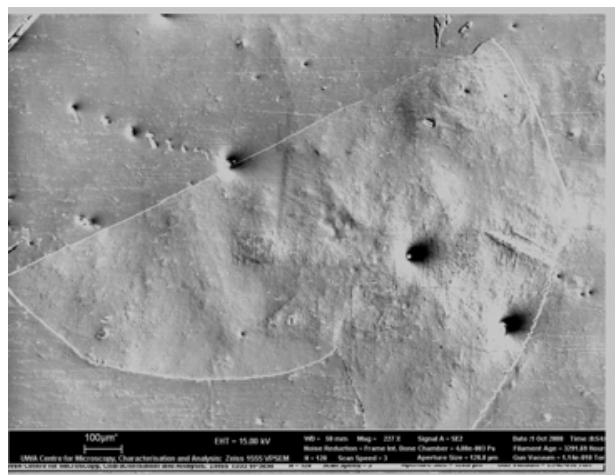

Figure 4: 50mm WD E-T SED after subtraction of an inverted, scaled BSE image. It matches closely fig.1(a)

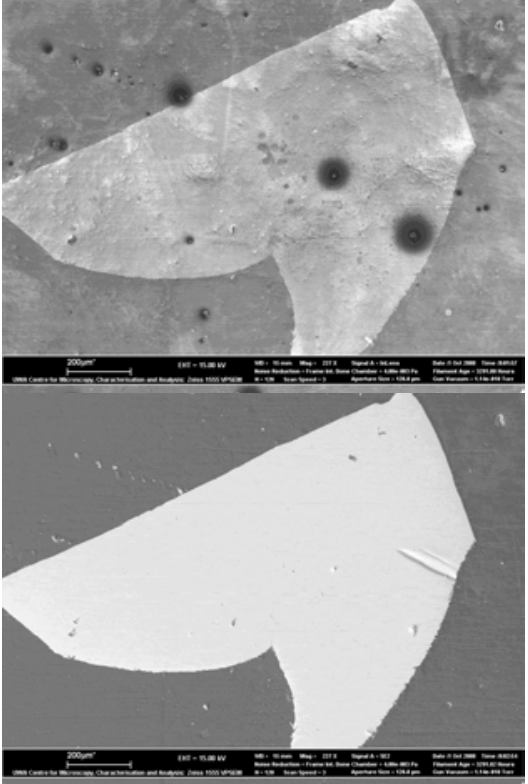

Figure 1(b): In-lens (top) and E-T (bottom) SE images of a polished $\mathrm{Cu}$ microprobe standard embedded in epoxy resin. $\mathrm{E}_{\mathrm{o}}=15 \mathrm{kV}$. Surface contamination and charging is visible in the INL SEI.

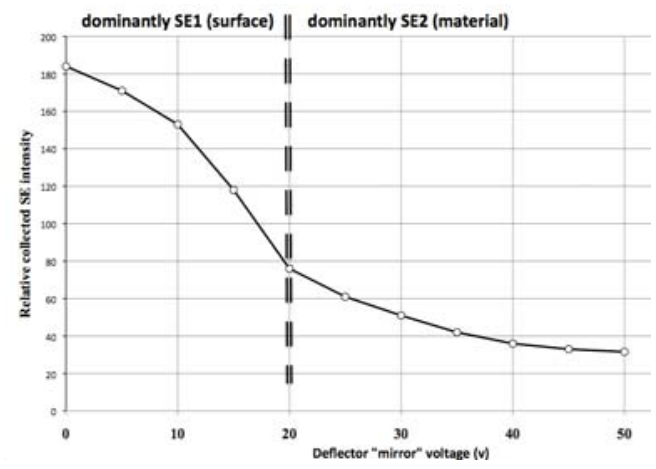

Figure 3: Measured relative SE signal intensity variation with FEI TLD "mirror" voltage.

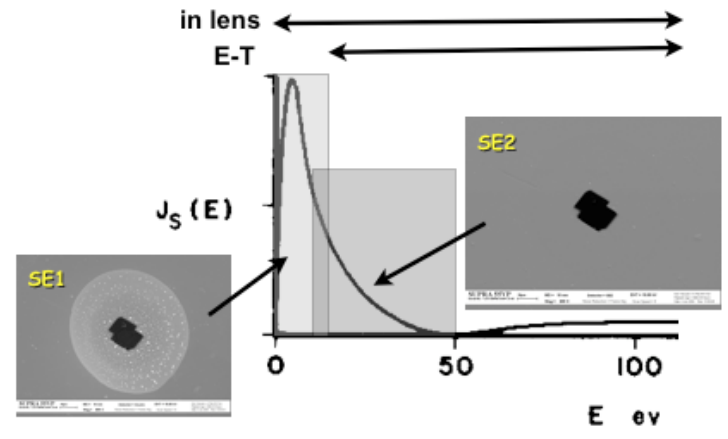

Figure 5: Schematic of SE1 and SE2 energy ranges for in lens and E-T SED. 